\title{
Reversible Acute Fontan Circulation Failure Secondary to Retroconducted Junctional Rhythm. Clinical Echocardiographic Correlation
}

Paolo Ferrero ( $\sim$ ferrerop41@gmail.com )

Aziende Socio Sanitarie Territoriale Papa Giovanni XXIII https://orcid.org/0000-0001-7967-7417

Isabelle Piazza

ASST Papa Giovanni XXIII: Aziende Socio Sanitarie Territoriale Papa Giovanni XXIII

Youcef Sadou

ASST Papa Giovanni XXIII: Aziende Socio Sanitarie Territoriale Papa Giovanni XXIII

Matteo Ciuffreda

ASST Papa Giovanni XXIII: Aziende Socio Sanitarie Territoriale Papa Giovanni XXIII

\section{Case Report}

Keywords: Heart failure, pediatric echocardiography, Cardiac pacing, congenital heart disease

Posted Date: January 18th, 2021

DOI: https://doi.org/10.21203/rs.3.rs-143984/v1

License: (9) This work is licensed under a Creative Commons Attribution 4.0 International License.

Read Full License

Version of Record: A version of this preprint was published at Journal of Congenital Cardiology on April 15th, 2021. See the published version at https://doi.org/10.1186/s40949-021-00063-1. 


\section{Abstract}

Background: Sequential atrioventricular activation plays a critical role in the physiology of Fontan circulation. Although bradycardia is usually well tolerated, retroconducted junctional rhythm may acutely increase atrial pressure impairing cardiac output. Echocardiographic evaluation can reveal clues of this hemodynamic condition. The clinical impact of arrhythmic disturbance on the follow up of patients who had undergone total cavo-pulmonary connection is well recognized but the role of, transient periods of retroconducted junctional rhythm on the immediate post-operative course is less defined.

Case presentation: We describe two cases of acute Fontan circulatory failure due to postoperative retroconducted escaping junctional rhythm despite an adequate heart rate and circadian variation. The patients rapidly improved after atrial pacing, allowing discharge with a minimal dose of diuretic.

Conclusion: In the absence of any hemodynamic target, hearth rhythm should be systematically checked after TCPC irrespective of adequacy of heart rate. Likewise, efficiency of temporary atrial pacing should be granted and surgeons should have a low threshold for epicardial lead implantation.

\section{Background}

Fontan circulation critically depend on pulmonary resistances and atrial pressure. [1] It has been observed that during sinus rhythm blood predominantly flow during atrial relaxation. Furthermore loss of atrioventricular synchrony may lead to phasic increase of atrial pressure which is transmitted to the Fontana circuit. [2] Heart rhythm optimization is recognized as a pivotal component of the follow up in this group of patients, while the impact of even self limited period of junctional rhythm is less defined, particularly wether the heart rate trend is acceptable.

We describe two cases of acute heart failure, after fenestrated total cavo-pulmonary connection (TCPC) which resolved after $48 \mathrm{~h}$ of atrial pacing. We demonstrated acute changes of Fontan flow pattern during the two modality of pacing that strictly correlated with the clinical status.

\section{Case Description}

The first patient was a four years old girl with hypoplastic left heart syndrome. She previously had undergone neonatal Norwood-Sano palliation and bidirectional cavo-pulmonary connection at the age of three months. Due to progressive cyanosis the patient was scheduled for tricuspid valve repair and TCPC. Preoperatively the patient had a well-tolerated junctional rhythm with constant retroconduction with preserved heart rate excursions.

Weaning from bypass was uneventful and the patient was successfully extubated after 12 hours.

During the first postoperative week abdominal congestion, right pleural effusion (Fig 1A) and progressive desaturation were observed. Blood chemistry disclosed increased liver enzymes and low albumin. 
Transthoracic echocardiogram showed normal ventricular function and mild AV valve regurgitation. Color-Doppler interrogation of the Fontan conduit and sovrahepatic veins, disclosed phasic hepatopetal signal consistent with a reversal flow through the fenestration on time with atrial retroconduction (Fig 2). This observation was consistent with the evidence of giant 'atrial' wave one the jugular venous pressure tracing. Upon atrial pacing the reverse component of flow disappeared with a slight increase in aortic velocity time integral (VTI).

Clinically, these echocardiographic changes were accompanied by resolution of pleural effusion and progressive increase of oxygen saturation and albumin normalization during the following days (Fig 1B). Based on this findings she was scheduled for permanent atrial pacing owing to persisting junctional rhythm.

The second patient was a five years old child who had undergone neonatal systemic to pulmonary shunt and cavo-pulmonary connection at the age of 9 months, due to severe symptomatic Ebstein anomaly. TCPC was planned because of progressive desaturation and effort intolerance. Few hours after cardiopulmonary bypass weaning he developed hypotension and low cardiac output syndrome requiring inotropic and vasopressor support. ECG disclosed competitive retroconduted junctional rhythm. Function of systemic ventricle and atrioventricular valve were normal. Similarly to the first case there was phasic reversal flow through the fenestration that was no longer detected during atrial pacing. Theses flow pattern changes translated into VTI increase and resolution of lactacidemia, in the following 24 hours, consistently with stroke volume and peripheral perfusion improvement. Permanent pacemaker implantation was not needed as sinus rhythm recovered after 72 hours.

\section{Discussion}

Despite surgical technical improvement, arrhythmic burden still represent a significant complication affecting the follow up of Fontan patients. $[3,4]$ The effect of cavo-pulmonary anastomosis modification on the incidence of arrhythmias is controversial, in particular the extra cardiac total cavopulmonary connection does not obviate the occurrence of sinus node dysfunction. [4] The clinical impact of this condition is often underestimated as bradycardia is usually deemed to be well tolerated in the context of Fontan circulation, particularly in the presence of an escaping junctional rhythm. Junctional rhythm in patients with Fontan circulation, by increasing the atrial pressure blunts the passive driving force of the Fontan circuit. [2]

This cases provide the echocardiographic proof of the correlation between postoperative retroconducted junctional rhythm and acute Fontan failure, which was completely reversible after atrial pacing.

It should be underscored that atrial activity retroconduction, differently from dissociated junctional rhythm, by generating a rhythmic abnormal increase of atrial pressure, is one of the the most important pathogenic determinants responsible for Fontan circulation failure in this clinical setting. 


\section{Conclusion}

In the absence of any hemodynamic target, hearth rhythm should be systematically checked after TCPC irrespective of adequacy of heart rate. Likewise, efficiency of temporary atrial pacing should be granted and surgeons should have a low threshold for epicardial lead implantation.

\section{Declarations}

\section{Ethical Approval and Consent to participate: NA}

Consent to participate and for publication : verbally requested and obtained

Availability of supporting data: available

Competing interest: none

\section{Funding: none}

Authors' contributoion: Dr Ferrero contributed to research design, analysis and interpretation of data; he drafted the paper and approved the submitted and final versions.

Dr Piazza contributed to research acquisition and interpretation of data; she drafted the paper and approved the final version.

Dr Sadou contributed to research acquisition and analysis of data; he revised critically the paper and approved the final version.

Dr Ciuffreda contributed to research design and interpretation of data; he revised critically the paper and approved the final version.

Acknowledgement: none

Authors' information: Dr Ferrero and Dr Ciuffreda work as pediatric cardiologist at Papa Giovanni XXIII Hospital in Bergamo - Italy. Dr Sadou works as neonatologist in Neonatal intensive care unit at Papa Giovanni XXIII Hospital in Bergamo, Italy. Dr Piazza is a Emergency medicine resident at University of Milan and works at Papa Giovanni XXIII Hospital in Bergamo - Italy.

\section{References}

1. Rychik J, Fogel MA, Donofrio MT, et al. Comparison of patterns of pulmonary venous blood flow in the functional single ventricle heart after operative aortopulmonary shunt versus superior cavopulmonary shunt. Am J Cardiol. 1997;80:922-26.

2. Hasselman T, Schneider D, Madan N, Jacobs M. Reversal of fenestration flow during ventricular systole in Fontan patients in junctional or ventricular paced rhythm. Pediatr Cardiol. 2005;26:638- 
41.

3. Hakacova N, Lakomy M, Kovacikova L. Arrhythmias after Fontan operation: comparison of lateral tunnel and extracardiac conduit. J Electrocardiol. 2008;41:173-7.

4. Stamm C, Friehs I, Mayer JE Jr, et al. Long-term results of the lateral tunnel Fontan operation. J Thorac Cardiovasc Surg 2001;121:28-41.

5. Cohen MI, Bridges ND, Gaynor JW, et al. Modifications to the cavopulmonary anastomosis do not eliminate early sinus node dysfunction. J Thorac Cardiovasc Surg 2000;120:891-900.

\section{Figures}

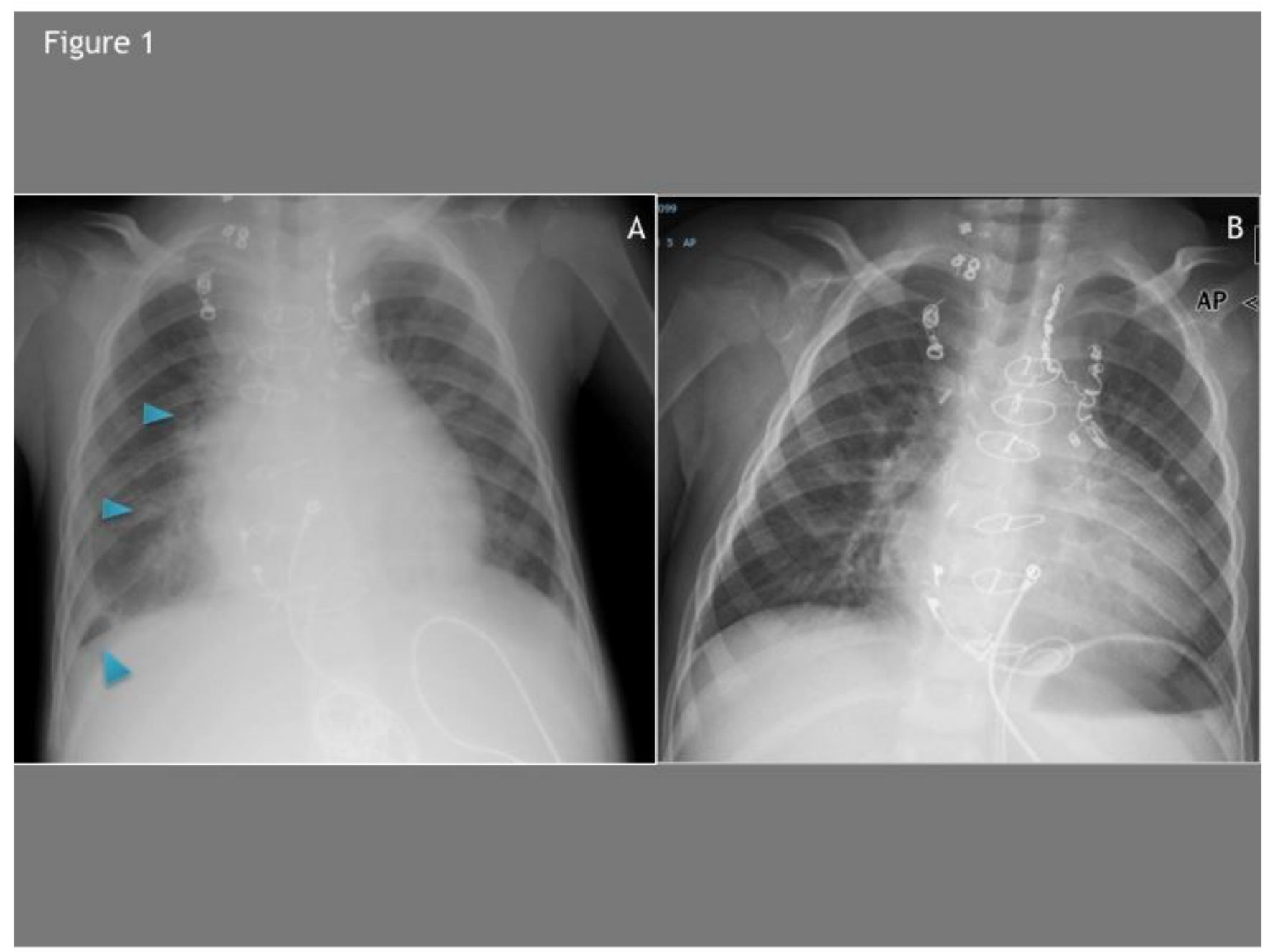

\section{Figure 1}

Postoperative chest $\mathrm{x}$-ray showing mild venous congestion and right pleural effusion (A) and normalization after $48 \mathrm{~h}$ atrial pacing (B). 


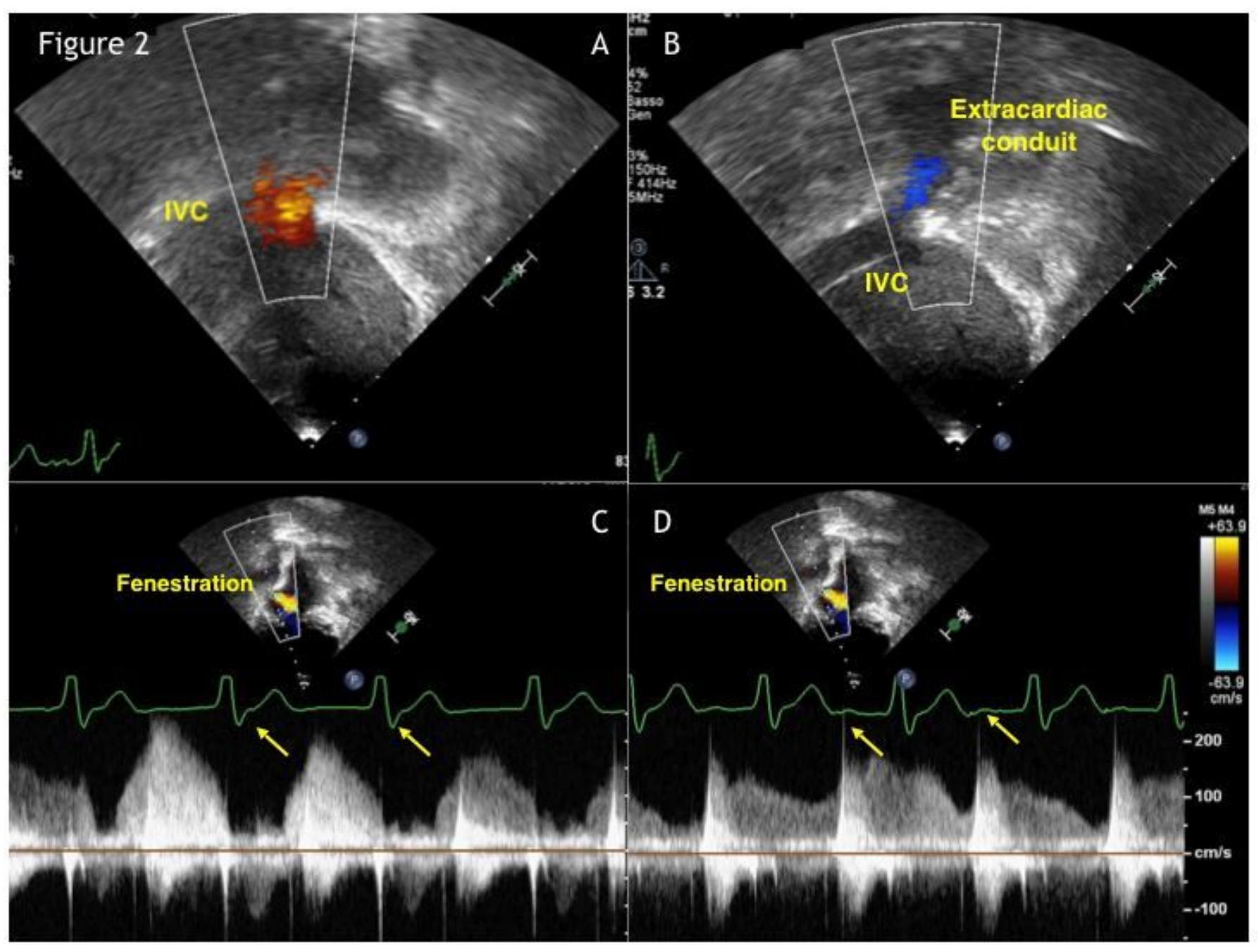

\section{Figure 2}

Echocardiographic frame showing phasic inversion (A) and continuous (B) flow in the Fontan con-duit during junctional rhythm and atrial paced rhythm, respectively (arrow). Pulse wave Doppler demonstrating inversion (C) and continuous (D) flow across the fenestration, during junctional and atrial paced rhythm, respectively. IVC: inferior vena cava 